\title{
CHARACTERIZATIONS OF NORMAL STRUCTURE
}

\author{
TECK-CHEONG LIM ${ }^{1}$
}

\begin{abstract}
The notions of asymptotic center for a decreasing net of sets and asymptotic normal structure are defined and several characterizations of normal structure are proved. Among these, the problem of whether complete normal structure is equivalent to normal structure is answered in the affirmative.
\end{abstract}

1. Introduction. Belluce and Kirk [1] first proved that if $K$ is a nonempty weakly compact convex subset of a Banach space and if $K$ has complete normal structure, then every family of commuting nonexpansive self-maps on $K$ has a common fixed point. Later, Holmes and Lau [6] extended this theorem to left reversible topological semigroups of nonexpansive self-maps (with some additional continuity assumption) of such a set $K$. In [7], the author proved, without showing the equivalence of normal structure and complete normal structure, that Belluce and Kirk's theorem holds for $K$ which is weakly compact and assumed only to have normal structure. However, the method used in [7] is not directly applicable to improving Holmes and Lau's result. In the present paper, we shall extend further the ideas contained in [7] and prove, as a corollary, that complete normal structure is equivalent to normal structure for weakly compact convex sets in Banach spaces (more generally, for weakly compact sets in locally convex Hausdorff linear topological spaces). A slightly neater proof of Holmes and Lau's theorem is then given.

2. Preliminaries and notations. Throughout this paper, $E$ will denote a separated, locally convex linear topological space and $Q$ a (fixed) family of continuous seminorms which generate the topology of $E$. For any $p \in Q$ and $A \subseteq E$, the symbol $\delta_{p}(A)$ will denote the $p$-diameter of $A$, that is

Presented to the Society, June 261973 ; received by the editors July 2, 1973.

AMS (MOS) subject classifications (1970). Primary 47H10, 46B99.

Key words and phrases. Normal structure, asymptotic center, asymptotic normal structure, complete normal structure, nonexpansive mapping, fixed point, topological semigroup.

${ }^{1}$ Part of this research was conducted while the author held an Izaak Walton Killam Memorial Scholarship at Dalhousie University under the supervision of Professor Michael Edelstein; it was also prepared while the author was at the Séminaire de Mathématiques Supérieures (Fixed point theory and its application), Université de Montréal.

(c) American Mathematical Society 1974 
$\delta_{p}(A)=\sup \{p(x-y): x, y \in A\}$, Co $A$ will denote the convex hull of $A$. $B_{p}(x, r)\left(B_{p}[x, r]\right)$ will denote the open (closed) ball with center at $x$ and $p$-radius $r$. If $x_{1}, \cdots, x_{n} \in E, \bar{x}_{n}$ will denote the element $n^{-1} \sum_{i=1}^{n} x_{i}$. For $A \subseteq E, \operatorname{cl}(A)$ will denote the closure of $A$.

If $H, K$ are nonempty subsets of $E, H$ being bounded, then for each $p \in Q$, we define:

$$
\begin{aligned}
r_{p}(H, x) & =\sup \{p(x-y): y \in H\}, \\
r_{p}(H, K) & =\inf \left\{r_{p}(H, x): x \in K\right\}, \\
\mathscr{C}_{p}(H, K) & =\left\{x \in K: r_{p}(H, x)=r_{p}(H, K)\right\} .
\end{aligned}
$$

Let $K$ be a convex subset of $E$. We say that $K$ has normal structure with respect to $Q$ ([1], [6], [8]) if, given any bounded (closed) convex subset $W$ of $K$ which contains more than one point, then, for every $p \in Q$ with $\delta_{p}(W)>0$, there exists $x \in W$ such that $\sup \{p(x-y): y \in W\}<\delta_{p}(W)$ or, equivalently, $\mathscr{C}_{p}(W, W)$ is a proper subset of $W$.

Let $K$ be a nonempty bounded closed convex subset of $E$. We say that $K$ has complete normal structure ([1], [6]) with respect to $Q$, if every closed convex subset $W$ of $K$ which contains more than one point and every $p \in Q$ with $\delta_{p}(W)>0$ satisfy: For any decreasing net $\left\{W_{\alpha}: \alpha \in A\right\}$ of nonempty subsets of $W$ with the property that $r_{p}\left(W_{\alpha}, W\right)=r_{p}(W, W)$ for all $\alpha \in A$, it is the case that the closure of $\bigcup\left\{\mathscr{C}_{p}\left(W_{\alpha}, W\right): \alpha \in A\right\}$ is a nonempty proper subset of $W$.

Let $C$ be a nonempty subset of $E$ and $\left\{W_{\alpha}: \alpha \in A\right\}$ be a decreasing net of nonempty bounded subsets of $E$. For each $x \in C$, each $\alpha \in A$ and each $p \in Q$, define

$$
\begin{gathered}
r_{p, \alpha}(x)=\sup \left\{p(x-y): y \in W_{\alpha}\right\}, \\
r_{p}(x)=\inf \left\{r_{p, \beta}(x): \beta \in A\right\} \text { and } r_{p}=\inf \left\{r_{p}(x): x \in C\right\} .
\end{gathered}
$$

The set $\left\{x \in C: r_{p}(x)=r_{p}\right\}$ (the number $r_{p}$ ) will be called the asymptotic center (asymptotic radius) of $\left\{W_{\alpha}: \alpha \in A\right\}$ in $C$ with respect to $p$ and denoted by $\mathscr{A}_{\mathscr{C}_{p}}\left(\left\{W_{\alpha}: \alpha \in A\right\}, C\right)\left(\operatorname{ar}_{p}\left(\left\{W_{\alpha}: \alpha \in A\right\}, C\right)\right)$. This definition is a generalization of that in [4], [5] and [7].

A convex set $C$ of $E$ is said to have asymptotic normal structure with respect to $Q$ if, given any bounded (closed) convex subset $W$ of $C$ which contains more than one point, given any decreasing net of nonempty subsets $\left\{W_{\alpha}: \alpha \in A\right\}$ of $W$ and given any $p \in Q$ such that $\delta_{p}(W)>0$, the asymptotic center of $\left\{W_{\alpha}: \alpha \in A\right\}$ in $W$ with respect to $p$ is a proper subset of $W$.

Some basic properties of $r(x)$ and asymptotic centers are collected as follows:

1. For each $x \in C$ and each $p \in Q,\left\{r_{p, \alpha}(x): \alpha \in A\right\}$ is a decreasing net in $R$ with limit $r_{p}(x)$. (A net $\left\{x_{\alpha}: \alpha \in \Lambda\right\}$ in $R$ is called decreasing if $x_{\alpha} \leqq x_{\beta}$ whenever $\alpha \geqq \beta$.)

2. For each $p \in Q, r_{p}(x)=0$ if and only if $\left\{W_{\alpha}: \alpha \in A\right\} \rightarrow^{p} x$, i.e. for every 
ball $B_{p}(x, r)$ of $x$ with $r>0$, there exists an $\alpha$ such that $W_{\alpha} \subseteq B_{p}(x, r)$.

3. For each $p \in Q,\left|r_{p}(x)-r_{p}(y)\right| \leqq p(x-y)$ for every $x, y \in A$. This follows from (1) and the fact that $\left|r_{p, \alpha}(x)-r_{p, \alpha}(y)\right| \leqq p(x-y)$ for every $\alpha \in A$.

4. Suppose that $C$ is convex. Then $r_{p}(x)$ is a continuous convex function on $C$ for every $p \in Q$. This follows from (3), (1) and the fact that $r_{p, \alpha}(x)$ are convex functions on $C$ for all $\alpha \in A$.

5. Suppose that $C$ is convex. Then, for each $p \in Q$, the asymptotic center of $\left\{W_{\alpha}: \alpha \in A\right\}$ in $C$ with respect to $p$ is a closed convex subset of $C$. This follows from (4).

6. If $C$ is weakly compact convex, then for each $p \in Q$, the asymptotic center of $\left\{W_{\alpha}: \alpha \in A\right\}$ in $C$ with respect to $p$ is nonempty. This follows from (4), the equality $\left\{x \in C: r_{p}(x)=r_{p}\right\}=\bigcap_{n=1}^{\infty}\left\{x \in C: r_{p}(x) \leqq r_{p}+1 / n\right\}$ and that closed convex subsets of weakly compact sets are weakly compact.

It is easy to see that the notion of asymptotic normal structure defined in this paper is an extension of that defined earlier by the author in [7].

A topological semigroup is a set $S$ together with an associative binary operation and a Hausdorff topology such that, for each $a \in S$, the two mappings from $S$ into $S$ defined by $s \rightarrow a s$ and $s \rightarrow s a$ for all $s \in S$, are continuous. $S$ is said to be left (right) reversible if any two (and hence any finite number) nonempty closed right (left) ideals of $S$ have nonempty intersection (cf. [3, p. 34]). An action of a topological semigroup $S$ on a topological space $X$ is a mapping $\psi$ from $S \times X$ into $X$ denoted by $\psi(s, x)=$ $s(x)$ such that $\left(s_{1} s_{2}\right)(x)=s_{1}\left(s_{2}(x)\right)$ for all $s_{1}, s_{2} \in S, x \in X$. The action is separately continuous if $\psi$ is continuous in each of the variables when the other is held fixed. If $X$ is a subset of $E$, then an action of $S$ on $X$ is nonexpansive if, for each $s \in S$, the mappings from $X$ into $X$ defined by $x \rightarrow s(x)$ for all $x \in X$ is nonexpansive with respect to $Q$, that is, $p(s(x)-s(y)) \leqq p(x-y)$ for all $p \in Q$ and $x, y \in X$.

3. Characterizations of normal structure. Throughout this and the next section, the notions of normal structure, complete normal structure, asymptotic normal structure and nonexpansive mappings are always taken with respect to $Q$.

The following two lemmas are straightforward generalizations of the corresponding versions in Banach space; proofs are omitted (see [2] for Lemma 1 and [7] for Lemma 2).

Lemma 1. A convex subset of $E$ has normal structure if and only if it does not contain a bounded sequence $\left\{x_{n}\right\}_{n \geqq 1}$ such that, for some $p \in Q$ with $\delta_{p}\left(\left\{x_{n}\right\}\right)>0$,

$$
d_{p}\left(x_{n+1}, \operatorname{Co}\left(x_{1}, \cdots, x_{n}\right)\right) \rightarrow \delta_{p}\left(\left\{x_{n}\right\}\right) \text { as } n \rightarrow \infty,
$$

where for $A \subseteq E, d_{p}(x, A)=\inf \{p(x-y): y \in A\}$. 
LEMMA 2. A convex subset of $E$ has normal structure if and only if it does not contain a bounded sequence $\left\{x_{n}\right\}_{n \geqq 1}$ such that, for some $p \in Q$ with $\delta_{p}\left(\left\{x_{n}\right\}\right)>0$ and some real number $c>0$,

$$
p\left(x_{n}-x_{m}\right) \leqq c \text { and } p\left(x_{n+1}-\bar{x}_{n}\right) \geqq c-1 / n^{2}
$$

for all $n \geqq 1, m \geqq 1$.

In what follows, we shall use $r_{p, \alpha}(x), r_{p}(x)$ and $r_{p}$ as defined in the previous section without referring to the original net $\left\{W_{\alpha}: \alpha \in A\right\}$ and set $W$ if no ambiguity can arise. The proof of the following theorem is analogous to that of Proposition 1 in [7]; we offer a sketch of it since this theorem is the central part of this paper.

THEOREM 1. A convex subset $C$ of $E$ has normal structure if and only if it has asymptotic normal structure.

Proof. The sufficiency part follows from the definition of asymptotic normal structure by taking $W_{\alpha}=W$ for each $\alpha \in A$.

For the necessity part, suppose that $C$ does not have asymptotic normal structure; let $W$ be a bounded convex subset of $C,\left\{W_{\alpha}: \alpha \in A\right\}$ a decreasing net of nonempty subsets of $W$ and $p$ a seminorm in $Q$ with $\delta_{p}(W)>0$, such that $r_{p}(x)=r_{p}$ for every $x \in W$. By basic property (2) following the definition of asymptotic normal structure, we have $r_{p}>0$. Let $x_{1}$ be an arbitrary element of $W$. Since $r_{p}\left(x_{1}\right)=r_{p}$, there exists an $\alpha \in A$ and an $x_{2} \in W_{\alpha}$ such that $p\left(x_{1}-x_{2}\right) \geqq r_{p}-1$. By moving $x_{2}$ towards $x_{1}$ along the line segment joining $x_{1}$ and $x_{2}$ if necessary, we may assume that $p\left(x_{1}-x_{2}\right) \leqq r_{p}$. Suppose that $x_{1}, x_{2}, \cdots, x_{n}$ have been chosen such that $x_{i} \in W, p\left(x_{i}-x_{j}\right) \leqq r_{p}, i=1, \cdots, n, j=1, \cdots, n$, and $p\left(x_{n}-\bar{x}_{n-1}\right) \geqq$ $r_{p}-1 /(n-1)^{2}$. We proceed to choose $x_{n+1}$ as follows: Since $A$ is a directed set and $r_{p}\left(x_{i}\right)=r_{p}, r_{p}\left(\bar{x}_{n}\right)=r_{p}$ for every $i=1, \cdots, n$, we can find an $\alpha \in A$ such that $r_{p, \alpha}\left(x_{i}\right) \leqq r_{p}+1 / n^{2}(n+1), \quad i=1, \cdots, n$, and $r_{p, \alpha}\left(\bar{x}_{n}\right) \leqq r_{p}+$ $1 / n^{2}(n+1)$. Choose $z_{0} \in W_{\alpha}$ such that $r_{p, \alpha}\left(\bar{x}_{n}\right)-p\left(z_{0}-\bar{x}_{n}\right) \leqq 1 / n^{2}(n+1)$. Let $z_{1}, \cdots, z_{n}$ be defined recursively by the formulae

where

$$
z_{i}=t_{i} x_{i}+\left(1-t_{i}\right) z_{i-1}, \quad i=1, \cdots, n,
$$

$$
t_{i}=\max \left(\frac{p\left(x_{i}-z_{i-1}\right)-r_{p}}{p\left(x_{i}-z_{i-1}\right)}, 0\right) \text { for each } i=1, \cdots, n .
$$

We then define $x_{n+1}=z_{n}$. An argument similar to that used in the proof of Proposition 1 in [7] shows that $p\left(x_{n+1}-x_{i}\right) \leqq r_{p}$ for all $i=1, \cdots, n$, $x_{n+1} \in W$ and that $p\left(x_{n+1}-\bar{x}_{n}\right) \geqq r_{p}-1 / n^{2}$. By induction, a sequence 
$\left\{x_{n}\right\}_{n \geqq 1}$ is constructed in $W$ such that the condition in Lemma 2 is satisfied with $c=r_{p}>0$. This implies that $C$ does not have normal structure and the proof is complete.

Corollary 1. A weakly compact convex subset $C$ of $E$ has normal structure if and only if it has complete normal structure.

Proof. The sufficiency part follows from the definition of complete normal structure by taking $W_{\alpha}=W$ for each $\alpha \in A$. Suppose that $W \subseteq C$ is a closed convex set which contains more than one point and $\left\{W_{\alpha}: \alpha \in A\right\}$ is a decreasing net of subsets of $W$ such that for some $p \in Q$ with $\delta_{p}(W)>0$, $r_{p}\left(W_{\alpha}, W\right)=r_{p}(W, W)$ for every $\alpha \in A$ and $\operatorname{cl}\left(\bigcup\left\{\mathscr{C}_{p}\left(W_{\alpha}, \mathrm{W}\right): \alpha \in A\right\}\right)$ is equal to $W$ (weak compactness of $W$ guarantees that $\bigcup\left\{\mathscr{C}_{p}\left(W_{\alpha}, W\right): \alpha \in A\right\}$ is nonempty, see [1]). Then clearly $r_{p}(x)=r_{p}(W, W)$ for every $x \in$ $\bigcup\left\{\mathscr{C}_{p}\left(W_{\alpha}, W\right): \alpha \in A\right\}$ so that, by continuity, $r_{p}(x)=r_{p}(W, W)$ for every $x \in W$. This, together with Theorem 1, proves the corollary.

4. Asymptotic centers and nonexpansive mappings. Suppose $X$ is a topological space and $S$ is a left reversible topological semigroup acting on $X$ such that the mapping $(s, x) \rightarrow s(x)$ is separately continuous. Then $S$ becomes a directed set if we define $a \geqq b$ if and only if $a S \subseteq \operatorname{cl}(b S)$ (see [6]). Moreover, if for a fixed element $u \in X$, we define $W_{s}=\operatorname{cl}(s S(u))$ for all $s \in S$, then the family $\left\{W_{s}: s \in S\right\}$ is a decreasing net of subsets of $X$ (see [6]). We have the following

THEOREM 2. Let $X$ be a nonempty, weakly compact convex subset of $E$. Let $S$ be a left reversible topological semigroup of nonexpansive, separately continuous, actions on $X$. Let $p \in Q$. Then the asymptotic center $A$ of the net $\left\{W_{s}: s \in S\right\}$ in $X$ with respect to $p$ is a nonempty, closed convex subset of $X$ and is mapped into itself by each element of $S$. If, moreover, $X$ has normal structure and $\delta_{p}(X)>0$, then $A$ is a proper subset of $X$.

Proof. By Theorem 1 and materials in $\S 2$, we have only to prove that $A$ is mapped into itself by each element of $S$. Let $x \in A$ and $s \in S$. Let $\varepsilon>0$ be arbitrary. Since $x \in A$, there exists $t \in S$ such that $t S(u) \subseteq W_{t} \subseteq$ $B_{p}\left[x, r_{p}+\varepsilon\right]$. Since $s$ is nonexpansive, we have $s t S(u) \subseteq B_{p}\left[s(x), r_{p}+\varepsilon\right]$ so that $W_{s t} \subseteq B_{p}\left[s(x), r_{p}+\varepsilon\right]$. It follows that $s(x) \in A$ and the proof is complete.

The following theorem is a consequence of Theorem 2 and is also a consequence of Corollary 1 in this paper, together with Theorem 1 in [6].

THEOREM 3. Let $K$ be a nonempty, weakly compact convex subset of $E$ and assume that $K$ has normal structure. Let $S$ be a left reversible topological semigroup of nonexpansive, separately continuous actions on $K$. Then $K$ 
contains a common fixed point for $S$, i.e. there exists $x \in K$ such that $s(x)=x$ for every $s \in S$.

Proof. Using Zorn's lemma, we obtain a subset $X$ of $K$ which is minimal with respect to being nonempty, closed, convex and mapped into itself by each element of $S$. Theorem 2 implies that $X$ is a singleton and hence contains a common fixed point of $S$.

Let $\left\{x_{\alpha}: \alpha \in \Lambda\right\}$ be a bounded net in $E$ and $C$ a nonempty subset of $E$. For each $\alpha \in \Lambda$, define $W_{\alpha}=\left\{x_{\beta}: \beta \geqq \alpha, \beta \in \Lambda\right\}$. The asymptotic center of $\left\{W_{\alpha}: \alpha \in \Lambda\right\}$ in $C$ with respect to a $p \in Q$ will be called, simply, the asymptotic center of $\left\{x_{\alpha}: \alpha \in \Lambda\right\}$ in $C$ with respect to $p$.

THEOREM 4. Let $X$ be a nonempty, weakly compact subset of $E$ and let $\mathscr{F}$ be a family of nonexpansive self-maps of $X$ such that every finite subfamily of $\mathscr{F}$ has a common fixed point in $X$. Let $\Lambda$ be the set of all finite subsets of $\mathscr{F}$, ordered by inclusion. Let $p \in Q$. Then we have:

(a) For each $\alpha \in \Lambda$, define $x_{\alpha}$ to be a common fixed point of $\alpha$. Then the asymptotic center $A$ of the net $\left\{x_{\alpha}: \alpha \in \Lambda\right\}$ in $X$ with respect to $p$ is a nonempty closed convex subset of $X$ which is mapped into itself by every member of $\mathscr{F}$. If, moreover, $X$ has normal structure and $\delta_{p}(X)>0$, then $A$ is a proper subset of $X$.

(b) For each $\alpha \in \Lambda$, define $W_{\alpha}$ to be the set of common fixed points of $\alpha$. Then the asymptotic center $A$ of the net $\left\{W_{\alpha}: \alpha \in \Lambda\right\}$ in $X$ with respect to $p$ is a nonempty closed convex subset of $X$ which is mapped into itself by every member of $\mathscr{F}$. If, moreover, $X$ has normal structure and $\delta_{p}(X)>0$, then $A$ is a proper subset of $X$.

Proof. (a) Let $x \in A$ and $f \in \mathscr{F}$. Let $\varepsilon>0$ be an arbitrary positive number. There exists $\alpha \in \Lambda$ such that $p\left(x_{\beta}-x\right) \leqq r_{p}+\varepsilon$ for all $\beta$ with $\beta \geqq \alpha$. If we let $\gamma=\{f\} \cup \alpha$, then for all $\beta \geqq \gamma$, we have

$$
p\left(f\left(x_{\beta}\right)-f(x)\right)=p\left(x_{\beta}-f(x)\right) \leqq p\left(x_{\beta}-x\right) \leqq r_{p}+\varepsilon .
$$

It follows that $f(x) \in A$. The rest of (a) follows from Theorem 1 and materials in $\S 2$.

The proof of part (b) is parallel to that of part (a) and is omitted.

Theorem 2 (and hence Theorem 1) in [7] follows from the above theorem by using a standard argument of Zorn's lemma (see the proof of Theorem 3 in this paper).

I thank Professor Hilton V. Machado for calling my attention to Theorem 4(a).

\section{REFERENCES}

1. L. P. Belluce and W. A. Kirk, Nonexpansive mappings and fixed points in Banach spaces, Illinois J. Math. 11 (1967), 474-479. MR 35 \#5988. 
2. M. S. Brodskir and D. P. Mil'man, On the center of a convex set, Dokl. Akad. Nauk SSSR (N.S.) 59 (1948), 837-840. (Russian) MR 9, 448.

3. A. H. Clifford and G. B. Preston, The algebraic theory of semigroups. Vol. I, Math. Surveys, no. 7, Amer. Math. Soc., Providence, R.I., 1961. MR 24 \#A2627.

4. M. Edelstein, The construction of an asymptotic center with a fixed-point property, Bull. Amer. Math. Soc. 78 (1972), 206-208.

5. - Fixed point theorems in uniformly convex Banach spaces (to appear).

6. R. D. Holmes and A. T. Lau, Non-expansive actions of topological semigroups and fixed points, J. London Math. Soc. (2) 5 (1972), 330-336.

7. T. C. Lim, A fixed point theorem for families of nonexpansive mappings, Pacific J. Math. (to appear).

8. K. K. Tan, Common fixed point theorems for almost weakly periodic nonexpansive mappings, Proc. Amer. Math. Soc. 33 (1972), 355-360. MR 45 \#4237.

Department of Mathematics, Dalhousie University, Halifax, Nova Scotia, CANADA 